\title{
INFORMATIZAÇÃO DOS INSTRUMENTOS DE AVALIAÇÃO DA MANUTENIBILIDADE PARA HOMOLOGAÇÃO DE SISTEMAS CONSTRUTIVOS INOVADORES ${ }^{1}$
}

\author{
INFORMATIZATIONOF MAINTAINABILITY EVALUATION INSTRUMENTS \\ FOR INNOVATIVE CONSTRUCTION SYSTEMS CERTIFICATION
}

\author{
Lucas Melchiori Pereira \\ Instituto de Arquitetura e Urbanismo, USP \\ Ic.melchiori@gmail.com \\ Nivaldo H. N. Bondança \\ Instituto de Arquitetura e Urbanismo, USP \\ hick209@gmail.com \\ Márcio Minto Fabrício \\ Instituto de Arquitetura e Urbanismo, USP \\ marcio@sc.usp.br \\ Daniel C. Reis \\ Instituto de Arquitetura e Urbanismo, USP \\ dreis7@gmail.com
}

\begin{abstract}
Resumo
A indústria do Ambiente Construído apresenta um histórico de pouca inovação tecnológica em parte devido à falta de garantias de qualidade do sistema ou componente desenvolvido durante todo ciclo de vida da edificação com ele construído. Um dos passos fundamentais para modificar essa realidade é garantir que tecnologias inovadoras sejam certificadas segundo requisitos dos usuários para operação, uso e manutenção. Neste sentido o Subprojeto 5 - Meta 45 do projeto INOVATEC - FINEP desenvolveu uma metodologia de avaliação da manutenibilidade de sistemas inovadores que visa reparar a falta de critérios precisos para avaliação deste tema observada nos sistemas de homologação vigentes. Alinhado a essa pesquisa, um trabalho consequente foi realizado com o objetivo de sistematizar, operacionalizar e refinar os instrumentos de coleta e organização dos indicadores de manutenção necessários para a avaliação de sistemas construtivos inovadores por meio da informatização dos formulários propostos. O artigo apresenta os resultados parciais desse trabalho, conceituando os instrumentos criados e analisando o desenvolvimento de sua informatização. Os resultados obtidos indicam além da contribuição inerente à informatização dos instrumentos, o potencial representado pelo desenvolvimento de aplicações simples para auxiliar métodos e processos do Ambiente Construído.
\end{abstract}

Palavras-chave: Instrumentos de Avaliação da Manutenibilidade. Homologação de Sistemas Construtivos Inovadores. Informatização dos instrumentos de avaliação da Manutenibilidade.

1PEREIRA, L.1et al. Informatização dos instrumentos de avaliação da manutenibilidade para homologação de sistemas construtivos inovadores. In: ENCONTRO BRASILEIRO DE TECNOLOGIA DE INFORMAÇÃO E COMUNICAÇÃO NA CONSTRUÇÃO, 7., 2015, Recife. Anais... Porto Alegre: ANTAC, 2015. 


\begin{abstract}
The Built Environment industry has a history of little technological innovation partly due to lack of system of quality assurance or component developed throughout the building lifecycle with it built. One of the fundamental steps to change this reality is to ensure that innovative technologies are certified according user requirements for operation, use and maintenance. In this sense the Subproject 5 - Meta 45 INOVATEC project - FINEP has developed a methodology for evaluating the maintainability of innovative systems aimed at repairing the lack of precise criteria for assessing this issue observed in the existing approval systems. Aligned to this research, a sizeable work was carried out in order to systematize, operate and refine the instruments of collection and organization of maintenance indicators needed for the evaluation of innovative construction systems through computerization of the proposed forms. The article presents the partial results of this work, conceptualizing the servants instruments and analyzing the development of its computerization. The results indicate besides the contribution inherent in the computerization of the instruments, the potential represented by the development of simple applications to aid methods and processes of the Built Environment.
\end{abstract}

Keywords: Maintaining Evaluation Instruments. Innovations Building Systems approval. Informatization of Maintainability evaluation Instruments.

\title{
1 INTRODUÇÃO
}

A indústria do Ambiente Construído apresenta um histórico de pouca inovação tecnológica e, quando esta ocorre, geralmente visam à redução de custos e agilidade no processo construtivo (REZENDE, BARROS e ABIKO , 2002). De fato são comuns os casos em que uma tecnologia inovadora foi incorporada em detrimento de melhorias na manutenção das edificações produzidas, prejudicando sua realização (BONIN, 1998)durante seu uso. Neste sentido pesquisas associadas ao Subprojeto 5 - Meta 45 do projeto INOVATEC - FINEP, voltado ao uso e manutenção de tecnologias inovadoras no âmbito da habitação social, apresentam a necessidade de melhorar a interface usuário-produto inovador, além de promover parâmetros para desenvolvimento de tecnológicas inovadoras que apresentem maior manutenibilidade e melhor desempenho.

A necessidade de melhorar a interface usuário-produto inovador tangencia temas associados à formação de mão de obra, disponibilidade materiais e tecnologias, adaptabilidade e flexibilidade de produtos/sistemas inovadores (SANCHES, 2013). Em seu relatório de pesquisa pertencente ao projeto, lara Del'Arco Sanches (2013, p. 07)aponta que "falta um sistema de uniformização do processo de homologação de produtos/sistemas inovadores", uma vez que a avaliação deste produtos são realizadas por Instituições Técnicas de Avaliação (ITA) independentes, que "não seguem um padrão homogêneo de avaliação." (SANCHES, 2013, p. 07).Com base nesta constatação a pesquisa desenvolvida por Sanches (2013) estabeleceu uma metodologia de avaliação da manutenibilidade de sistemas construtivos baseados na consideração de requisitos dos usuários e a relevância da etapa de operação, uso e manutenção (SANCHES, 2013, p. 85). Com isso, pretende-se estabelecer um processo homogeneizado às instituições técnicas avaliadoras vinculadas ao SINAT. O método proposto, contudo, demandava maior refinamento e sistematização de instrumentos, consolidando modelos para fichas operacionais e informatizadas.

\subsection{Objetivo do Artigo}

A necessidade de operacionalizar estes instrumentos para avaliação e homologação de sistemas construtivos dependia da integração dos requisitos e instrumentos propostos em um sistema de tabulação interativo baseado em autonomação, de forma a permitir o incremento de novos requisitos sem que o sistema perca consistência informacional. Nas palavras de seu principal teorizador a autonomação é "uma forma de automação com um toque humano" (SHINGO, 1996). Trata-se de um conceito originado na Engenharia de Produção (EP) que preconiza o desenvolvimento de um sistema automático de controle de anormalidades. Originalmente empregado na concepção de máquinas, atualmente a autonomação é apontada no âmbito da Engenharia de Software (ES) como uma 
metodologia ou instrumento ágil para o Desenvolvimento de Software, permitindo que o sistema garanta consistência de informações incorporadas (CRESCÊNCIO, 2014).

A constituição do sistema de homologação da manutenibilidade de sistemas construtivos inovadores baseado em autonomação parte da necessidade de garantir leveza aos formulários e consistência aos diferentes instrumentos propostos. Assim, o objetivo principal da pesquisa é oferecer as bases informacionais para esse sistema autonomatizado. $E$ para realizar o objetivo principal é preciso refinar os instrumentos de avaliação para homologação de sistemas construtivos propostos na metodologia de avaliação de sistemas construtivos inovadores com foco na manutenção, desenvolvidas em pesquisas do projeto INOVATEC FINEP: Subprojeto 5 - Meta 45 (SANCHES, 2013), por meio da tabulação dos dados primários levantados, integração das correlações existentes entre os diferentes instrumentos propostos e sistematização destes dados em formulários operacionais e informatizados. $\mathrm{O}$ presente artigo contextualiza a pesquisa e exemplifica como ocorre a construção das estruturas necessárias para a realização da informatização dos instrumentos.

\section{REVISÃO BIBLIOGRÁFICA}

A característica multidisciplinar da pesquisa resulta em uma revisão heterogênea, que deve apresentar tanto as bases para formulação dos instrumentos de avaliação de tecnologia inovadoras como os meios utilizados para informatização desses instrumentos. Assim nas subseções seguintes serão apresentados os conceitos de inovação tecnologia e manutenção, o que é o sistema de homologação dessas tecnologias, como a avaliação da manutenibilidade se insere nesse sistema e como os formulários de avaliação são compostos, finalmente são apresentados os fundamentos e ferramentas adotados para a sistematização dos formulários de avaliação.

\subsection{Inovações tecnológicas}

Empresas de diferentes segmentos desenvolvem projetos inovadores motivados por vários objetivos, como melhoria de produtos, maior eficiência e qualidade, ou ainda, capacidade de aprendizado e implementação de mudanças (OECD, 1997). As inovações podem envolver alterações que substituem inteiramente o processo convencional (HINO e MELHADO, 2001) - ou seja, uma Inovação radical (OECD, 1997) - como se restringir a alterações introduzidas ao processo existente. Neste ultimo caso, trata-se de um tipo de Inovação incremental (TERTRE e BAS, 1997) que resulta em um aumento no grau de industrialização do processo, aumentando o grau de racionalização, mecanização e pré-fabricação sem romper os limites de manufatura característicos do processo tradicional(SANCHES, FABRÍCIO e BUZZAR, 2015). Sob qualquer uma das perspectivas apresentadas, são tímidas as iniciativas em inovações tecnológicas na construção. Entre os fatores que interferem neste processo destacam-se as barreiras culturais a serem vencidas, calcadas na manutenção de práticas tradicionais de produção, bem como as barreiras governamentais, por meio de políticas insipientes de incentivo governamental à adoção de novas tecnologias, em sua maioria inadequada ou não fundamentada (TOLEDO; ABREU; JUNGLES, 2000; SANCHES; FABRÍCIO; BUZZAR, 2015).

Para a CAIXA (2011) o termo sistemas construtivos inovadores (SCI) engloba aqueles sistemas que incorporam uma nova concepção, que represente alternativas à tecnologia convencional, não seja objeto de norma brasileira prescritiva e não seja tradicionalmente utilizada no país. Alinhado a este conceito, o Programa Brasileiro de Qualidade e Produtividade no Habitat (PBQP-H) - Sistema Nacional de Avaliação Técnica (SINAT) visa harmonizar requisitos, critérios e métodos para avaliação técnica de produtos inovadores no Brasil, ordenando procedimentos para a concessão dos documentos de avaliação técnica. $O$ SINAT é uma iniciativa da comunidade técnica nacional que avalia produtos não 
abrangidos por normas técnicas prescritivas, suprindo assim as lacunas existentes na normalização técnica prescritiva (MINISTÉRIO DAS CIDADES, 2012).

\subsection{Manutenção}

Um dos aspectos fundamentais para a avaliação de tecnologias inovadoras, comumente desconsiderado ou restringido, é a observação de sua manutenibilidade. A NBR 5674:2012 - Manutenção de Edificações classifica essa prática em três categorias: Manutenção preventiva, Manutenção baseada na condição e manutenção corretiva. Durante o desenvolvimento de um projeto é comum o desenvolvimento de atividades associadas ao projeto, materiais e execução, esquecendo-se do planejamento e uso/manutenção (SANTOS, 2003). Trata-se de um equívoco recorrente em muitas normas e adotado por profissionais, que ignoram a necessidade de garantir a Manutenibilidade de uma construção.

Manutenibilidade é "uma característica intrínseca ao equipamento que permite um maior grau de facilidade na execução dos serviços de manutenção" (ROSA, 2006, p. 58). Além disso, o projeto dos serviços de manutenção precisa incorporar informações ligadas aos custos de capital, da segurança e confiabilidade dos equipamentos, custos operacionais e de manutenção. Estudos mostram que 8 a $10 \%$ das falhas nas construções têm origem na etapa de utilização, e que o custo da manutenção pode representar 1,5 a $2 \%$ do valor total da obra, em apenas cinco anos (SANTOS, 2003, p. 50). Estes indicativos apontam para uma relação estreita entre a manutenibilidade e o desempenho da edificação.

A norma NBR 15575: Edificações Habitacionais - Desempenho (ABNT, 2013), reafirma a ligação entre desempenho, durabilidade e manutenção, ao apontar "a maior facilidade ou dificuldade de manutenção e reparação em caso de falha no desempenho" (ABNT, 2013). Esta norma estabelece ainda uma divisão de responsabilidades entre os intervenientes envolvidos: construtoras, moradores, condomínios. Um ponto fundamental relacionada à divisão de responsabilidades relaciona-se ao usuário e sua interação com a qualidade da edificação. Neste sentido a observação da NBR 14037 - Manual de Operação, Uso e Manutenção das Edificações (ABNT, 1998) além da NBR ISO 9000 - Sistemas de gestão da qualidade - Fundamentos e vocabulário (ABNT, 2005) e ISO 9001 - Sistemas de gestão da qualidade - Requisitos oferecem um importante subsidio para analisar se um sistema construtivo inovador contempla considerações suficientes para a etapa de operação, uso e manutenção de forma a proporcionar desempenho satisfatório e atendimento aos requisitos do usuário(SHIMBO e MARTINS, 2015).

\subsection{Sistemas de homologação}

Internacionalmente, existem diversas instituições responsaveis por sistemas de homologação por meio da avaliação técnica de produtos inovadores. Algumas dessas instituições se organizam em comunidades "com o objetivo de harmonizar e padronizar parâmetros de metodologias de avaliação e requisitos" (SANCHES, 2013). Alguns exemplos significativos de instituições em Portugal, Dinamarca e Espanha, estabelecem relações com mais de uma comunidade além da Comunidade Europeia, de que seus países fazem em parte. Particularmente na Comunidade Europeia os padrões harmonizados de avaliação para produtos inovadores são estabelecidos por comissões de padrões, que se baseiam em procedimentos pré-estabelecidos na Directive 83/189/EEC (EUROPEAN PARLIAMENT, 2003). Essa diretiva geral visa cumprimento de requisitos essenciais para a construção, estabelecendo o conteúdo mínimo para a aprovação técnica (EUROPEAN PARLIAMENT, 2003), denominada European Technical Approval (ETA): Lista dos documentos interpretativos pertinentes; Requisitos específicos para os produtos na acepção dos requisitos essenciais; Procedimentos para teste; Métodos de avaliação e de avaliação do resultado dos testes; Procedimentos de inspeção e de conformidade que devem 
corresponder a disposições do documento Directive 83/189/EEC; Período de validade do documento ETA.

Os requisitos essenciais devem compreender uma vida útil economicamente razoável e satisfazer as condições normais de uso e manutenção. Abrangem resistência mecânica e estabilidade; segurança contra incêndio; higiene, saúde e ambiente; segurança no uso; proteções contra ruído e; economia de energia e retenção de calor. Outra iniciativa apara harmonizar e facilitar a troca de informações e experiências sobre procedimentos e escopo utilizados nas avaliações técnicas em diversos países é o World Federation of Technical Assessment Organization - WFTAO: uma rede internacional composta por 21 países: África do Sul, Austrália, Brasil, Canadá, Dinamarca, Espanha, Estados Unidos, França, Finlândia, Hungria, Israel, Itália, Japão, Noruega, Nova Zelândia, Polônia, Portugal, Reino Unido, República Tcheca, Romênia, Rússia (WFTAO, 2012). Semelhante ao observado na Comunidade Europeia, com o ETA, outros países espalhados pelo mundo possuem seus próprios métodos e documentação de avaliação. Estes, porem, alinham-se as orientações da comunidade WFTAO para avaliação e homologação de sistemas inovadores (WFTAO, 2012).

\subsection{Instrumentos de avaliação da manutenibilidade de sistemas construtivos inovadores - SINAT}

Instituto de Pesquisas Tecnológicas de São Paulo, o IPT, representa o Brasil na WFTAO. Sua atuação na homologação de produtos inovadores na Construção Civil ocorre por meio do Sistema Nacional de Avaliações Técnicas de Produtos Inovadores.

O processo de homologação de sistemas inovadores segue quatro etapas estabelecidas. A primeira etapa inicia-se com a empresa proponente do produto inovador, que deve elaborar a solicitação de avaliação técnica de seu produto. Então, uma Instituição Técnica Avaliadora (ITA) elabora uma minuta de diretrizes para este produto, que deve ser avaliado por um comitê técnico e encaminhado para a comissão nacional referendar as diretrizes que serão publicadas pela Secretaria Geral (SANCHES, 2013). A segunda etapa corresponde ao Relatório Técnico de Avaliação (RTA), onde, após a publicação das diretrizes, a ITA realiza ensaios para avaliar o desempenho do produto inovador. Caso o resultado seja satisfatório a ITA realiza a auditoria inicial da qualidade do processo de produção. Se a auditoria for satisfatória, a ITA inicia a terceira etapa, com a elaboração de uma minuta do Documento de Avaliação Técnica (DATec). Então, um comitê técnico analisa essa minuta e a encaminha para comissão nacional conceder a chancela SINAT ao DATec. Chancelado, cabe a Secretaria Geral comunicar a ITA. A quarta e última etapa do sistema de homologação do SINAT inicia-se com a publicação do DATec. Cabe a ITA a responsabilidade de publicá-lo e encaminhá-lo à Secretaria Geral e ao proponente. A Secretaria Geral então confere e arquiva o DATec no banco de dados e a empresa proponente do produto inovador autoriza o controle periódico através de auditorias técnicas definidas no DATec.

Os DATecs possuem uma estrutura padrão para todos os documentos que incorpora uma descrição do produto ou sistema construtivo inovador, suas condições e as limitações de uso, a diretriz para avaliação técnica, informações e dados técnicos, resultados resumidos da avaliação técnica, aspectos do controle da qualidade, fontes de informação consideradas e condições de emissão (SINDUSCON, 2011). Embora exista uma clara evolução na estrutura dos DATecs atuais quando comparados aos primeiros realizados, observa-se ainda nestes documentos uma abordagem insuficiente à questões de manutenibilidade. Por exemplo, com relação às condições e limitações de uso, a maioria dos DATecs restringemse a informar que os cuidados na utilização devem constar nos Manuais de Operação, Uso e Manutenção e que esses devem ser preparados pelas empresas proponentes dos sistemas inovadores avaliados em questão (SANCHES, 2013). De fato, baseando-se em um 
levantamento onde todos os 18 documentos de homologação publicados até então foram analisados, Sanches (2013) aponta que apenas dois DATecs abordam a assistência técnica, "um mais especificamente falando da etapa de pós-entrega da edificação e outro mencionando o termo "condições de assistência técnica" (SANCHES, 2013, p. 46).

Como a implantação de novas tecnologias (produtos e processos) na construção do Ambiente Construído (AC) visa obter melhorias globais nos índices de desempenho, produtividade, diminuição de desperdícios e de tempo (REZENDE, BARROS e ABIKO , 2002), a manutenção acaba por sofrer pouca alteração neste processo, inclusive sofrendo certo retardo (BONIN, 1998).Porém, ao menos parcialmente, os SCls apresentam tecnologias novas e desconhecidas, o que exige maior cuidado com fatores ligados à operação, uso e manutenção. Entende-se que a manutenção deve acompanhar o dinamismo do processo e das alterações de necessidades, interesses e aspirações dos usuários além das crescentes exigências de qualidade ambiental junto ao desenvolvimento tecnológico e social (SANCHES, 2013). Assim, a homologação de SCI deve garantir uma orientação suficiente tanto às etapas de concepção e projeto na adoção de materiais, tecnologias e SCl, para que estas considerem adequadamente as implicações desta adoção na etapa de operação, uso e manutenção de forma a proporcionar desempenho satisfatório e atendimento aos requisitos do usuário. Estas condições estão relacionadas às possibilidades do sistema construtivo inovador permitir interface com outros sistemas construtivos e como elas acontecem, bem como à disponibilidade da tecnologia ou knowhow necessário para gestão do sistema e disponibilização de assistência técnica para a etapa de uso pelos produtores.

A Meta 45 do projeto INOVATEC - FINEP apresenta subsídios para superar a deficiência observada nos DATecs em avaliar a manutenibilidade dos $\mathrm{SCl}$, através de instrumentos da metodologia de avaliação estruturados adequadamente, de maneira a proporcionar desempenho satisfatório e atendimento aos requisitos do usuário. Os instrumentos desenvolvidos para subsidiar a avaliação contemplam cinco formulários sendo um realizado antes da homologação e quatro a ser realizado após a liberação do DATec, onde será avaliado o desempenho da tecnologia inovadora no uso e manutenção da construção. Desta forma o modelo considera uma validação posterior a homologação, em que o DATec é avaliado em uso, conforme é apresentado no Quadro 1.

Quadro 1- Fases da avaliação da manutenibilidade de tecnologias inovadoras

\begin{tabular}{|l|l|l|}
\hline Fase & Descrição dos instrumentos \\
\hline PRÉ-DATec & Roteiro de avaliação da manutenibilidade com base em análise de \\
& documentos técnicos; & \\
\hline PÓS-DATec & $\bullet$ & Entrevista com síndico/zelador \\
& Entrevista com profissional de assistência técnica \\
& - Questionário com o usuário \\
& Roteiro de inspeção para avaliação da manutenção \\
\hline
\end{tabular}
Fonte: baseado em Sanches (2013, p. 76)

Cada instrumento relacionado no Quadro 1 apresenta um conjunto de questões ligadas a um requisito de manutenibilidade. Ao todo são 408 itens a serem observados, entre questões objetivas e entrevistas orientadas, sendo 185 questões objetivas do "Roteiro de avaliação da manutenibilidade com base em análise de documentos técnicos", 34 tópicos para entrevista estruturada da "Entrevista com síndico/zelador", 12 tópicos para entrevista estruturada da "Entrevista com profissional de assistência técnica", 38 questões objetivas do "Questionário com o usuário" e 139 questões do "Roteiro de inspeção para avaliação da manutenção".

Cabe destacar que, dos 408 itens, 362 são questões objetivas. Cada uma dessas questões 
objetivas solicita por respostas avaliações qualitativas registradas de forma padronizada, o que oferece uma base quantitativa, passível de ser mensurada. Associada a essas respostas objetivas, são associadas informações auxiliares, como medição, observação do avaliador competente e registro fotográfico que ilustre a situação ou anomalia eventualmente identificada.

As respostas objetivas atentam para o atendimento normativo e como este ocorre. Ou seja, o requisito deve ser qualificado segundo o atendimento a norma de desempenho e de manutenção (quando estas existirem), indicando se atende, não atende ou atende parcialmente. Além disso, é preciso avaliar o desempenho observado em cada requisito de manutenibilidade, conforme indicado no Quadro 2.

Trata-se de um conjunto de informações consideráveis que precisam ser tabulados de maneira a expressar uma síntese numérica. Este trabalho, significativamente trabalhoso se feito manualmente, é automaticamente realizado em um sistema informatizado de registro, armazenamento e computação dos resultados da avaliação.

Quadro 2 - classificação do estadode conservação observado

- Atende às normas de manutenção e desempenho;

- Permite que sejam feitos reparos, reformas e ampliações;

- No caso de reparos, reformas e ampliações podem ser utilizadas tanto o sistema construtivo inovador quanto sistemas construtivos tradicionais;

- A mão de obra não precisa ser especializada ou é disponibilizada pela empresa responsável pelo sistema construtivo inovador;

- Os materiais necessários à manutenção e às reformas e ampliações encontra-se disponível no mercado;

- A tecnologia necessária é disponibilizada pela empresa responsável pelo sistema construtivo inovador no caso de reparos, reformas ou ampliações.

\begin{tabular}{ll} 
Normal: $\quad \begin{array}{l}\text { Atende às normas de manutenção e desempenho permitindo que sejam } \\
\text { feitos apenas reparos e desempenho de serviços de manutenção; }\end{array}$ \\
\hline
\end{tabular}

Mínimo;

Deficiente;

Inexistente.

Fonte: Autores (2015).

\subsection{Sistema ágil e Autônomo}

O desenvolvimento de um sistema informatizado dos instrumentos "Roteiro de inspeção para avaliação da manutenção", "Roteiro de avaliação da manutenibilidade com base em análise de documentos técnicos"; e "Questionário com o usuário" oferece desafios relacionados à necessidade de permitir a fácil adição de questões por ITAs responsáveis pelas RTAs; versatilidade e leveza no funcionamento; possibilidade de atualizar código fonte durante uso do sistema; funcionamento online e off-line. Essas características são atendidas adequadamente pelo JSON (Java Script Object Notation), uma Notação de Objetos Java Script cuja formatação leve de troca de dados alia a facilidade em de ler e escrever, para o programador, à facilidade em interpretar e gerar para os programas que reconhecem a linguagem (ECMA INTERNATIONAL, 2011).

O JSON "é em formato texto e completamente independente de linguagem, pois usa convenções que são familiares às linguagens $\mathrm{C}$ e familiares, incluindo $\mathrm{C}++, \mathrm{C \#}$, Java, Java Script, Perl, Python e muitas outras"(JSON.ORG, 2003) que o faz um formato interessante para troca de dados. O JSON baseia-se em um subconjunto da linguagem de programação 
Java Script, Standard ECMA-262 3a Edição -Dezembro - 1999 (ECMA INTERNATIONAL, 2011), constituído fundamentalmente por duas estruturas: uma coleção de pares nome/valor (name/value) - que pode ser object, record, strut, dictionary, hash table, keyed list ou associative array - e uma lista ordenada de valores. Como essas duas estruturas são universais, ou seja, são suportadas por todas as linguagens de programação(JSON.ORG, 2003). Isso permite que eventuais sistemas desenvolvidos por uma RTA se comuniquem facilmente ao sistema proposto. Ao mesmo tempo, a oferta do sistema básico para registro, armazenamento, comunicação e computação dos resultados da avaliação, permite a uniformização dos dados levantados pelas RTAs de diferentes instituições avaliadoras, consolidando bases rastreáveis e comparáveis entre os documentos que fundamentam os DATecs.

Embora a linguagem JSON apresente as facilidades de interatividade, sua interface é pouco amigável a usuários. Para que informações sejam inseridas e retiradas do JSON por pessoas sem conhecimento em programação de sua linguagem é preciso desenvolver um parsing (ROUSE, 2001), onde é realizada a análise sintática de uma sequência de entrada para determinar sua estrutura gramatical segundo uma gramática formal específica(MARCUS, 1978). Ou seja, um analisador sintático processa um texto de entrada, que pode ser lida de uma tabela Excel transformando-o em uma estrutura de dados que pode ser convertida para outra linguagem como, por exemplo, o JSON. O inverso deste processo também é possível, quando uma entrada sob a forma de código sequencial de programação pode ser quebrada em partes, organizado - por exemplo, em forma de substantivos (objetos), verbos (métodos) e seus atributos ou opções - e reordenado em um formato de leitura, como PDF (ROUSE, 2001).

\section{METODOLOGIA DE PESQUISA}

O enunciado do objetivo aponta que a pesquisa não visa apenas explicar os fenômenos relacionados aos instrumentos de avaliação propostos, mas principalmente apresentar soluções às questões reais associadas a sua operacionalização ao mesmo tempo em que contribui teoricamente ao entendimento das disciplinas na qual esse conhecimento é aplicado. Lukka (2003, p. 1) aponta a Pesquisa Construtiva, ou Constructive Research, como o caminho para uma pesquisa cientifica prescritiva como esta, que visa uma solução a dado problema real. Trata-se de um campo específico e emergente do conhecimento, cujo mérito metodológico se concentra no entendimento, modelagem e instrumentalização de artefatos humanos: Todos os artefatos humanos, tais como modelos, diagramas, planos, estruturas organizacionais, produtos comerciais e projetos de sistemas de informação são construções. Em comum, todos se caracterizam por serem inventados e desenvolvidos, em vez de descobertos. (LUKKA, 2003, p. 2)

Assim, o presente trabalho se alinha à pesquisa construtiva, pois visa à operacionalização dos instrumentos de avaliação propostos na metodologia de avaliação de sistemas construtivos inovadores com foco na manutenção (SANCHES, 2013). Após identificar o problema e obter uma compreensão teórica sobre o assunto foi preciso conceber e desenvolver o artefato / solução esperada. O artefato, no caso, um sistema informatizado para registro, armazenamento, computação e comunicação dos resultados da avaliação de manutenibilidade de tecnologias inovadoras, foi concebido em linguagem JSON. Para a execução da programação foi estruturada a arquitetura do sistema, esta etapa exigiu a revisão dos instrumentos originalmente desenvolvidos para simplificar e uniformizar a formatação das questões (ou objetos). Os instrumentos informatizados foram validados em pesquisa de campo onde foram validados tanto o sistema como a metodologia de avaliação propostas.

A seguir é reportado em resultados e discussões a construção do instrumento de "Roteiro de 
inspeção para avaliação da manutenção", que exemplifica a estruturação dos objetos do sistema e lista ordenada de valores. Maiores detalhes dessa pesquisa estão reportados no relatório final de pesquisa encaminhado à FINEP.

\section{RESULTADOS E DISCUSSÕES}

A análise formal dos instrumentos de avaliação de manutenibilidade propostos para o SINAT (SANCHES, 2013)revelaram que estes são constituídos por entrevista estruturada e questionário objetivo. Este último é composto por itens de verificação que contemplam os requisitos normativos de manutenibilidade. Cada item pode ser composto por duas questões além de um espaço para anexar observação e fotografia. A primeira questão aponta se o requisito em questão foi atendido e a segunda questão qualifica qual é o estado de conservação observado. Todos os resultados são tabulados para alimentar uma fórmula que os convertem em um índice geral de manutenibilidade.

Para que o trabalho de quantificação das respostas e cálculo do índice seja automatizado por meio da informatização, foi preciso estabelecer primeiramente uma base de dados de entrada, em formato Excel, contendo colunas para um código de ordenação e agrupamento dos itens, os tipos de questões envolvidas, a redação dos itens e a redação do texto de observação a serem expostos no aplicativo (Quadro 3).

Quadro 3 parte da planilha Excel de valores de entrada para o formulário

\begin{tabular}{|c|c|c|c|}
\hline COD & Tipo & Valores 1 (texto título, Questão) & Valores 2 (texto de obs.) \\
\hline 1 & & \multicolumn{2}{|l|}{ Ficha de Inspeção para Avaliação da Manutenção } \\
\hline 1.A & & \multicolumn{2}{|l|}{ vistoria de áreas internas } \\
\hline 1.A.1 & & \multicolumn{2}{|l|}{ Paredes/Painéis de Vedação } \\
\hline 1.A.1.1 & 1 & $\begin{array}{l}\text { As fissuras existentes são menores ou iguais aos valores } \\
\text { máximos estipulados para o sistema aplicado *usar } \\
\text { fissurômetro* }\end{array}$ & $\begin{array}{l}\text { Indicar as medidas das } \\
\text { fissuras }\end{array}$ \\
\hline 1.A.1.2 & 1 & $\begin{array}{l}\text { Paredes sem deformações e desaprumos e em } \\
\text { conformidade com as normas pertinentes }\end{array}$ & \\
\hline 1.A.1.3 & 1 & Paredes sem sinais de infiltração e manchas & \\
\hline 1.A.1.4 & 1 & Ortogonalidade de cantos (usar esquadro) & \\
\hline 1.A.1.5 & 2 & Estado de conservação * & \\
\hline 1.A.2 & & \multicolumn{2}{|l|}{ Estrutura } \\
\hline 1.A.2... & $\ldots$ & $\ldots$ & $\ldots$ \\
\hline
\end{tabular}

Fonte: Autores (2015).

Um parser foi desenvolvido para que os valores inseridos no arquivo Excel sejam convertidos para o formato Jason, preenchendo os valores indicados na tabela, conforme exemplificado no Quadro 4. O arquivo resultante é lido pela aplicação, que monta a interface do formulário seguindotemplates pré-definidos dos tipos de questão, que são completadose agrupadosorientados pela ordenação do "code", que identifica e hierarquia no formulário as partes, seçõese ítens de verificação. 
Quadro 4 - exemplo de formulário vazio e preenchido²

\begin{tabular}{|c|c|}
\hline Formulário vazio & Formulário preenchido \\
\hline 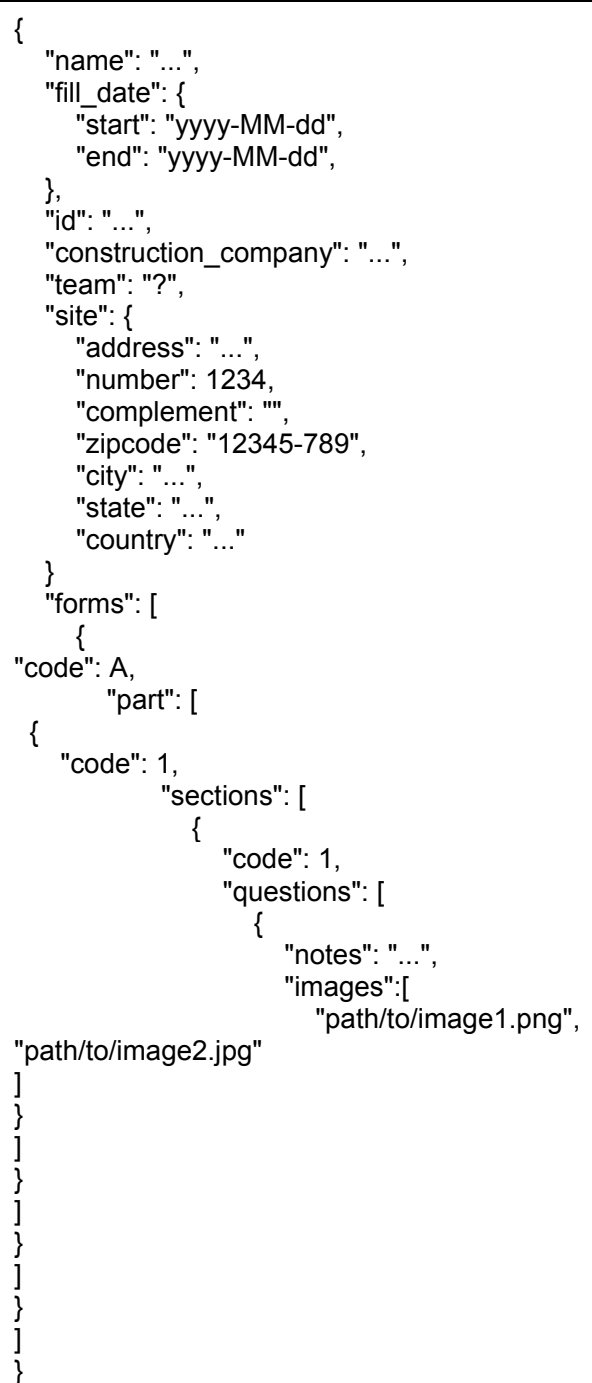 & 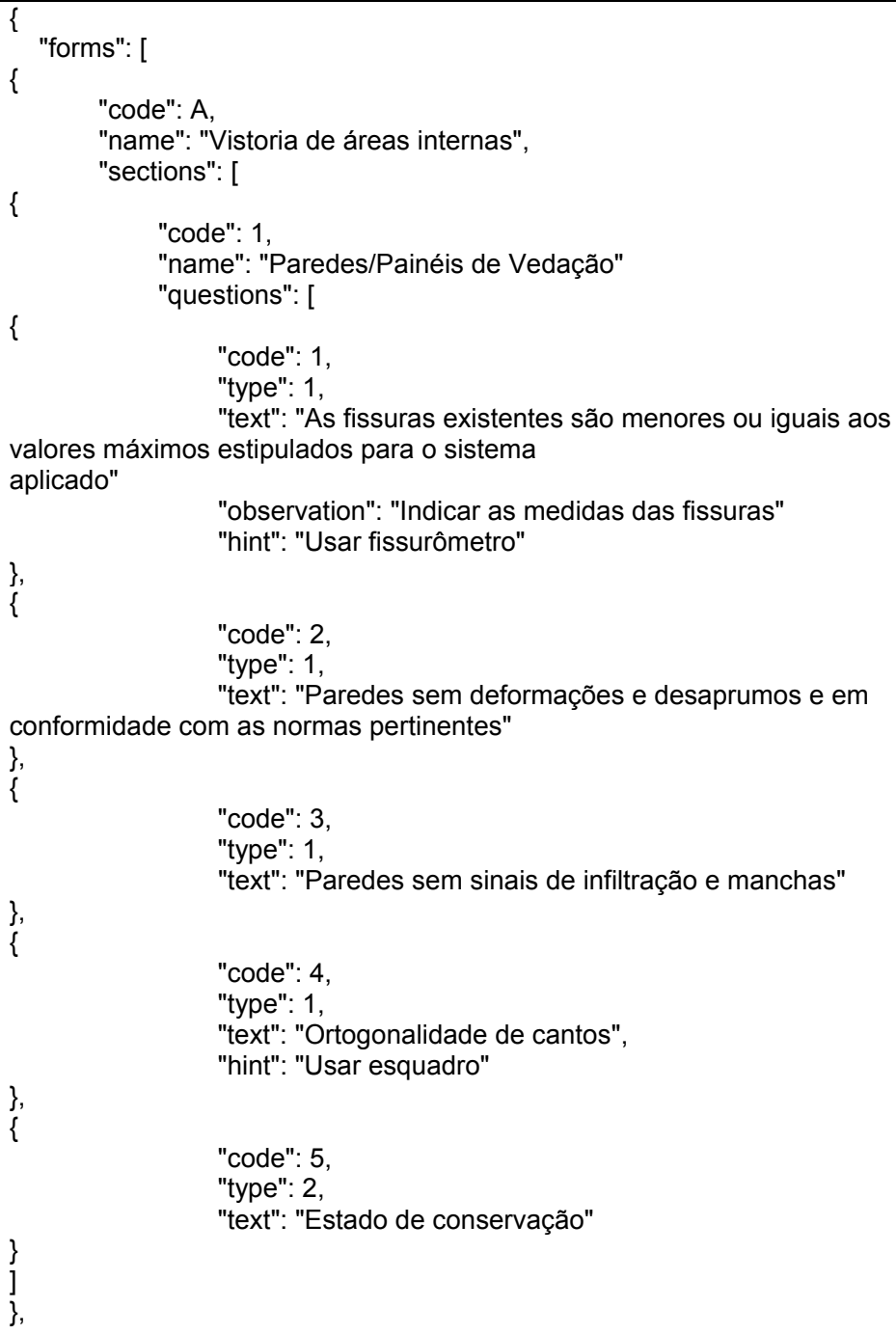 \\
\hline
\end{tabular}

Fonte: Autores (2015).

No exemplo de programaçãodo Formulário preenchido apresentado noQuadro 4, o "type" é indicado em uma lista numérica 1, 2 ou 3, que comunica ao aplicativo qual formato de resposta deve ser dada. Ou seja, ao indicar "type": 1 para questão de "code": 3 , o arquivo JSON indica ao aplicativo que a resposta para "Paredes sem sinais de infiltração e manchas" deve ter como resposta uma das variaveis apresentadas pela composição do tipo 01( 
Quadro 5) seguida pela solicitação de uma observação e indexação de figuras a esta resposta. Caso a formatação indicasse "type": 2, o formato de resposta seria condizente às variáveis apresentadas no Quadro $2^{3}$.

${ }^{3} \mathrm{~A}$ interface do aplicativo destinada ao avaliador está disponível no Google play e pode ser encontrado pesquisando pelo nome "Inovatec M45". 
Quadro 5 - composição do tipo 01

\begin{tabular}{|cc|}
\hline & "text":(buscar Texto título) \\
\hline [ ] atende (A) & [ ] não atende (NA) [ ] não aplica (NSA) \\
\hline "hint": (buscar texto observação) & \\
\hline
\end{tabular}

Fonte: Autores (2015).

Com base nas respostas registradas, são realizadas pelo aplicativo as métricas de cálculo do índice geral de manutenibilidade. Esses valores, assim como os dados primários do levantamento são exportados para um arquivo de formato JSON. Outro parser foi programado para converter estes dados exportados para uma interface mais amigavel, completando um template de relatório com resumo do índice, memorial de cálculo e gráficos, alem da relação das respostas, observações e fotos associadas aos itens de verificação levantados.

Cada interveniente tem um acesso específico aos registros gerados. $O$ avaliador, que gera o arquivo PDF, deve conferir o memorial de cálculo e reportar eventual incoerencia ao ITA, este pode editar a planilha Excel de valores de entrada para o formulário e gerar o JSON que será lido pelo aplicativo, porem não tem acesso à programação dos parsers e da aplicação. O SINAT, por sua vez, tem permissão para alterar e públicar online versões atualizadas da programação dos parsers e da aplicação, que são carregadas automaticamente nos aparelhos cadastrados pelo sistema, assim que estiverem conectados. Erros e sugestões observados durante a realização de uma avaliação devem ser reportados à coordenação SINAT, tambem os arquivos JSON de respostas devem ser encaminhados, de maneira a alimentar um bando de dados de todos os DATecs.

\section{CONCLUSÕES}

A adaptabilidade possível graças à arquitetura desenvolvida para o sistema permite a continua revisão dos formulários de manutenibilidade, de maneira que tanto o SINAT possa atualizar o corpo principal do formulário, atualizando-o online o conteúdo e forma de todas bases para RTAs utilizadas pelas ITAs, como as ITAs podem adicionar sem dificuldade questões pertinentes a uma determinada tecnologia em homologação a ser avaliada.

A sequência autônoma de transformações entre modelos de arquivos adequados ao manuseio do usuário, como Excel, e formatos JSON por meio de um PARSER, quando os formulários são gerados, bem como o caminho inverso, quando as informações registradas no aplicativo em formato JSON são convertidas para formatos seguros como Excel e PDF estão associadas a permissões especificas de alteração da programação para SINAT, ITAs e operadores. Estas barreiras informacionais são consideradas importantes para incolumidade e rastreabilidade dos dados levantados.

O desenvolvimento de uma rede de troca e integração de dados entre ITAs e SINAT, embora não realizado, foi considerado na formulação deste modelo básico de aplicativo. Ao incorporar o JSON como meio de comunicação dos dados dos formulários, o aplicativo proposto reflete a demanda por interoperabilidade entre diferentes sistemas envolvidos. A adoção de aplicações ágeis ainda é pouco empregada na indústria do Ambiente Construído.

A experiência observada neste trabalho é um exercício de aproximação entre as áreas de tecnologias do AC e a informática. Mesmo em pesquisas de rápida duração, como seis meses, é possível desenvolver e validar aplicações com testes em campo estruturados, relevando a relevância e consistência dos resultados alcançados. Estruturadas como pesquisa construtiva, novas pesquisas associadas ao desenvolvimento de aplicações oferecem uma oportunidade de pesquisa cientifica, desde que observada às orientações apresentadas na revisão, que é desenvolvida e amadurece dia a dia. 


\section{REFERÊNCIAS}

ABNT. NBR 14037: Manual de operação, uso e manutenção das edificacões - conteúdo e recomendações para elaboração e apresentação. Associação Brasileira de Normas Técnicas. Rio de Janeiro, p. 05. 1998.

ABNT. NBR ISO 9000: Sistemas de gestão da qualidade - Fundamentos e vocabulário. Associação Brasileira de Normas técnicas. Rio de Janeiro, p. 35. 2005.

ABNT. NBR 15575: Edificações Habitacionais - Desempenho. Partes 1à 5. Associação Brasileira de Normas Técnicas. Rio de Janeiro. 2013.

BONIN, L. C. Considerações sobre a utilização do conceito de desempenho como instrumento para a modernização tecnológica na construção de edificações. Encontro Nacional de Tecnologia do Ambiente Construído, 7. Florianópolis: [s.n.]. 1998.

CAIXA. Inovações Tecnológicas na CAIXA. Seminário: Sistemas Industrializados à Base de Cimento para Habitação. Material de apresentação, São Paulo, 2011.

CRESCÊNCIO, S. Lean. In: PRIKLADNICKI, R.; WILLI, R.; MILANI, F. Métodos Ágeis para Desenvolvimento de Software. Porto Alegre: Bookman, 2014. p. 102-118.

ECMA INTERNATIONAL. The JSON Grammar. In: INTERNATIONAL, E. ECMAScript Language. Geneva: Ecma International, 2011. p. 9.

EUROPEAN PARLIAMENT. The Construction Products Directive. Council Directive 89/106/EEC amended by Council Directive 93/68/EEC, 1993. Regulation (EC): № 1882/2003 of European Parliament and of the Council. 29 setembro 2003.

HINO, M. K.; MELHADO, S. B. Qualidade do projeto de empreendimentos habitacionais de interesse social: proposta utilizando o conceito de desempenho. Boletim Técnico da Escola Politécnica da USP, Departamento de Engenharia de Construção Civil, BT/PCC/303, São Paulo, 2001. 20.

HOLMSTRÖM, J.; KETOKIVI, M.; HAMERE, A.-P. Bridging Practice and Theory: A Design Science Approach. Decision Sciences, Finland, v. 40, n. Journal compilation, 2009, Decision Sciences Institute, p. 65 - 87, fevereiro 2009.

JSON.ORG. Introdução ao JSON. json, 01 mar. 2003. Disponivel em: <http://json.org/json-pt.html>. Acesso em: 13 abr. 2015.

MARCUS, M. P. A theory of syntactic recognition for natural language. Massachusetts Institute of Technology. Cambridge, p. 417. 1978. (http://hdl.handle.net/1721.1/16176).

MINISTÉRIO DAS CIDADES. Sistema Nacional de Avaliações - SINAT. PBQO-H, 2012. Disponivel em: <http://www.cidades.gov.br/pbqp-h/projetos_sinat.php>. Acesso em: 28 outubro 2014.

OECD. Manual de Oslo. Diretrizes para coleta e interpretação de dados sobre inovação. Gabinete Estatístico das Comunidades Europeias. Traduzido sob a responsabilidade da FINEP Financiadora de Estudos e Projetos, 2004. [S.I.], p. 184. 1997.

REZENDE, M. A. P. D.; BARROS, M. M. S. B. D.; ABIKO , K. Barreiras e facilitadores da inovação tecnológica na produção de habitações populares. in: Encontro Nacional de Tecnologia no Ambiente Construído Artigo Técnico, 9. Foz de Iguaçu. Anais: ENTAC. 2002. p. 895-904. disponivel em < http://www.iau.usp.br/pesquisa/grupos/arquitec/entac/2002/Artigos/ENTAC2002_0895_904.pdf >acesso em: 25/10/2014.

ROSA, E. B. Indicadores de desempenho e sistema ABC: o uso de Indicadores para uma gestão eficaz do custeio e das atividades de manutenção. Tese (Doutorado) - Escola Politécnica, Universidade de São Paulo. São Paulo, p. 530. 2006.

ROUSE, M. Parser Definition. searchsoa, 2001. Disponivel em:

<http://searchsoa.techtarget.com/definition/parser>. Acesso em: 10 maio 2015.

SANCHES,. Desenvolvimento de Métodos e Metodologias para Avaliação de Desempenho de Tecnologias Inovadoras no Âmbito do Sistema Nacional de Avaliação Técnica. Instituto de Arquitetura e Urbanismo - USP. São Carlos, p. Projeto INOVATEC - FINEP - Subprojeto 5:Uso e 
manutenção de tecnologias inovadoras no âmbito da habitação social - Meta 45. Relatório Final. 2013.

SANCHES, I. D.; FABRÍCIO, M. M.; BUZZAR, M. A. Avaliação da manutenibilidade em sistemas construtivos inovadores. In: FABRÍCIO, M. M.; ONO, R. Avaliação de Desempenho de Tecnologias Construtivas Inovadoras: manutenção e percepção dos usuários. Porto Alegre: Antac, 2015. Cap. 7, p. $67-87$.

SANTOS, A. D. O. Manual de operação, uso e manutenção das edificações residenciais: coleta de exemplares e avaliação de seu conteúdo frente às diretrizes da NBR 14.037/1998 e segundo as perspectivas dos usuários. Dissertação (Mestrado) - Universidade Federal do Rio Grande do Sul. Porto Alegre, p. 179. 2003.

SHIMBO, L. Z.; MARTINS, M. E. A questão da manutenção de sistemas construtivos inovadores para habitação. In: FABRÍCIO, M. M.; ONO, R. Avaliação de Desempenho de Tecnologias Construtivas Inovadoras. Porto Alegre: [s.n.], 2015. Cap. 6, p. 55 - 66.

SHINGO, S. O Sistema Toyota de Produção: do ponto de vista da Engenharia de Produção. Porto Alegre: Bookman, 1996.

SINDUSCON. Inovações e SINAT. Revista Notícias da Construção, São Paulo, p. 44-45, dezembro 2011.

TERTRE, C. D.; BAS, C. L. L'innovation et les entreprises à ingénierie intégrée dans le bâtiment.

Collection Recherche, Paris, v. recherche $\mathrm{n}^{\circ}$ 85, p. 190, maio 1997. ISSN 978-2-11-085575-6.

TOLEDO, R.; ABREU, A. F.; JUNGLES, A. E. A difusão de inovações tecnológicas na indústria da construção civil. in: Encontro Nacional de Tecnologia no Ambiente Construído Artigo Técnico, 8. Salvador. Anais: ENTAC. 2000. p. 317-324.

WFTAO. World Federation of Technical Assessment Organization.Disponível em: http://www.wftao.com/. Acesso em cinco de outubro de 2014. 2012. 